\title{
In vitro activity of isavuconazole against Candida auris by time-kill and
}

Unai Caballero ${ }^{1}$, Elena Eraso², Javier Pemán ${ }^{3}$, Guillermo Quindós² and Nerea Jauregizar ${ }^{1}$

1 Department of Pharmacology. Faculty of Medicine and Nursery, University of the Basque Country (UPV/EHU), Bilbao, Spain. 2 Department of Immunology, Microbiology and Parasitology. Faculty of Medicine and Nursery, UPV/EHU, Bilbao, Spain. (GIC15/78 IT-990-16, UFI 11/25). 3 Servicio de Microbiología, Hospital

Universitario y Politécnico La Fe, Valencia, Spain

unai.caballero@ehu.eus

\section{BACKGROUND AND OBJECTIVES}

Candida auris is an emerging species causing disseminated candidiasis associated with high mortality and has a challenging treatment due to its difficult diagnosis and a multidrug resistant profile, specially to azoles like fluconazole and voriconazole. Isavuconazole is a broad spectrum novel triazole agent that exerts fungistatic and fungicidal activities. It has been approved for the treatment of invasive aspergillosis and mucormicosis and it also shows activity against Candida.

The aim of this study was to study the in vitro activity of isavuconazole against Candida auris by time-kill (TK) methodology and postantifungal (PAFE) effect assays.

\section{MATERIALS AND METHODS}

In vitro static TK experiments and PAFE assays were performed against three Candida auris clinical blood isolates from the outbreak of candidiasis at the Hospital Universitario Politécnico La Fe (Valencia, Spain) (1) as previously described (2). TK and PAFE were carried out on microtiter plates in RPMI (final volume $200 \mu \mathrm{L}$ ) and using an inoculum of 1-5 CFU/mL. Based on minimum inhibitory concentration (MIC) values previously determined by EUCAST E.def 7.3 method (3), isavuconazole (Basilea, UK) concentrations tested for both TK and PAFE experiments were 0.06, 0.125, 0.25, 0.50 and $1 \mathrm{mg} / \mathrm{L}$. For PAFE assays, after the cells were exposed to isavuconazole for $1 \mathrm{~h}$, the drug was removed by a process of 3 cycles of repeated centrifugations (2000 rpm, $10 \mathrm{~min}$ ) and washed with PBS. For both experiments, samples for viable count quantification were obtained at $0,2,4,6,8,24$ and $48 \mathrm{~h}$ and inoculated onto a Sabouraud dextrose agar plate for CFU counting. Plots of averaged colony counts $\left(\log _{10} \mathrm{CFU} / \mathrm{ml}\right)$ versus time were constructed and compared against a growth control (in absence of drug). TK and PAFE experiments were performed simultaneously and in duplicate.

\section{RESULTS}

None of the concentrations of isavuconazole assayed in TK experiments showed significant antifungal activity, even though a concentration-dependent fungal growth reduction trend was observed when compared with the control curve. As it could be expected form TK results, no PAFE effect was detected against any of the studied isolates.
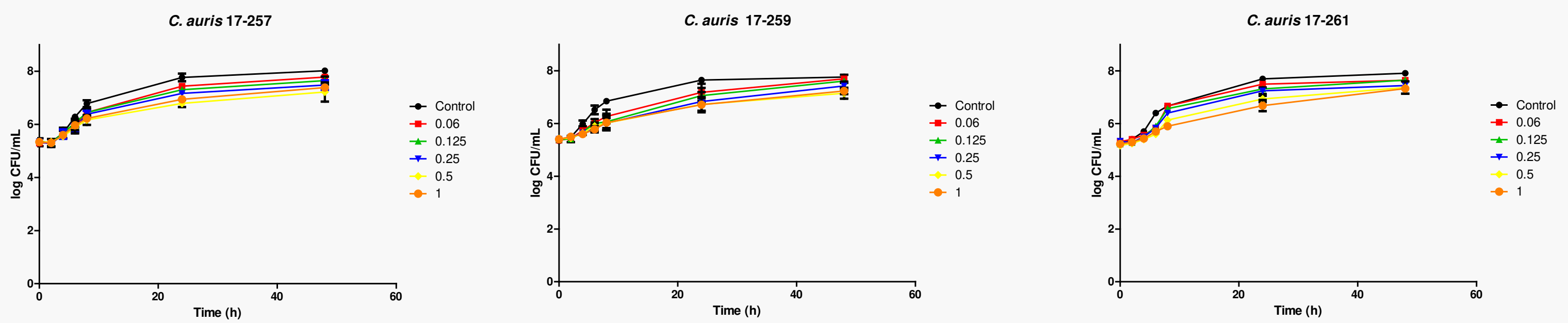

Figure 1. Mean TK curves for isavuconazole against Candida auris. Each data point represents the mean result \pm standard deviation.
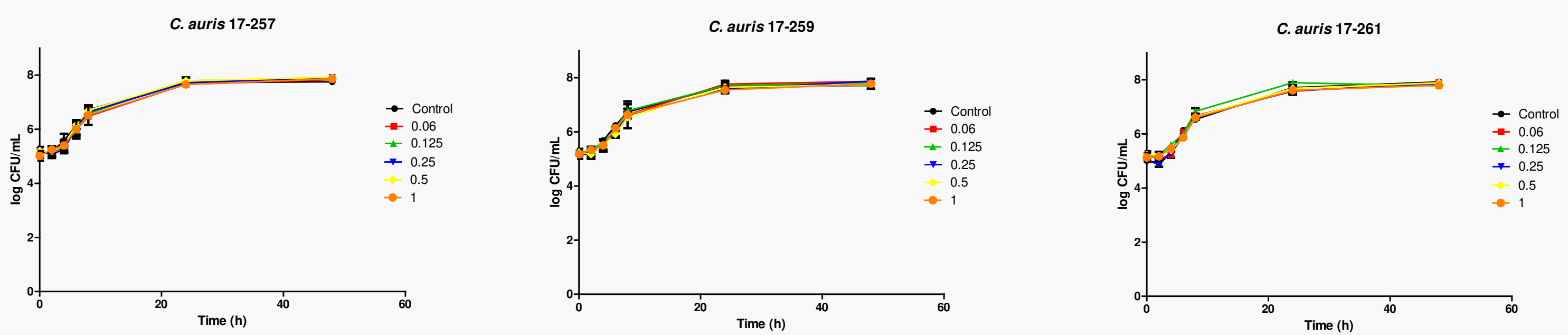

Figure 2. Mean PAFE curves for isavuconazle against Candia auris. Each data point represents the mean result \pm standard deviation.

\section{CONCLUSIONS}

Despite the low MICs, isavuconazole did not show significant activity against the studied Candida auris isolates in the performed TK experiments. These results suggest that special care should be taken when interpreting the MIC values for resistant species such as Candida auris and other in vitro methodologies like TK experiments should also be considered for a more complete characterization of antimicrobial activity. 